\title{
Regenerative and Rehabilitative Medicine: A Necessary Synergy for Functional Recovery from Volumetric Muscle Loss Injury
}

\author{
Sarah M. Greising ${ }^{\mathrm{a}}$ Christopher L. Dearth ${ }^{\mathrm{b}}$ Benjamin T. Corona ${ }^{\mathrm{a}}$ \\ a Extremity Trauma and Regenerative Medicine, US Army Institute of Surgical Research, Fort Sam Houston, Tex., and \\ ${ }^{b}$ DoD-VA Extremity Trauma and Amputation Center of Excellence, Walter Reed National Military Medical Center, \\ Bethesda, Md., USA
}

\section{Key Words}

Individualized medicine - Muscle graft - Orthopedic trauma .

Physical medicine $\cdot$ Physical therapy $\cdot$ Regenerative

medicine $\cdot$ Rehabilitation $\cdot$ Skeletal muscle injury $\cdot$ Tissue

engineering

\begin{abstract}
Volumetric muscle loss (VML) is a complex and heterogeneous problem due to significant traumatic or surgical loss of skeletal muscle tissue. The consequences of VML are substantial functional deficits in joint range of motion and skeletal muscle strength, resulting in life-long dysfunction and disability. Traditional physical medicine and rehabilitation paradigms do not address the magnitude of force loss due to VML and related musculoskeletal comorbidities. Recent advancements in regenerative medicine have set forth encouraging and emerging therapeutic options for VML injuries. There is significant potential that combined rehabilitative and regenerative therapies can restore limb and muscle function following VML injury in a synergistic manner. This review presents the current state of the VML field, spanning
\end{abstract}

The opinions or assertions contained here are the private views of the authors and are neither to be construed as official or as reflecting the views of the Department of the Army, the Department of Defense, nor the US Government.

\section{KARGER}

Published by S. Karger AG, Basel

E-Mail karger@karger.com

www.karger.com/cto clinical and preclinical literature, with particular focus on rehabilitation and regenerative medicine in addition to their synergy. Moving forward, multidisciplinary collaboration between clinical and research fields is encouraged in order to continue to improve the treatment of VML injuries and specifically address the encompassing physiology, pathology, and specific needs of this patient population. This is a work of the US Government and is not subject to
copyright protection in the USA. Foreign copyrights may apply.
Published by S. Karger AG, Basel

\section{Introduction}

High-energy blast trauma secondary to improvised explosive device use is one of the signature injury patterns suffered by US Servicemen during the military engagements in Operations Enduring Freedom, Iraqi Freedom,

\begin{tabular}{ll} 
Abbreviations used in this paper \\
\hline ECM & extracellular matrix \\
GFP & green fluorescent protein \\
TA & tibialis anterior \\
TEMR & tissue-engineered muscle repair \\
UBM & urinary bladder matrix \\
VML & volumetric muscle loss \\
\hline
\end{tabular}


and New Dawn. Recent advances in military medicine have resulted in dramatic improvements in combat casualty survival. As a result, a significant number of battlefield-injured military personnel has returned from combat with extensive extremity trauma injuries. These extremity trauma injuries often involve severe soft tissue injury and commonly result in persistent functional deficits and chronic disability. Of significant concern to prolonged functional outcomes is the volumetric loss of skeletal muscle (VML), which may result from the initial trauma (e.g., combat-related blast or ballistic trauma) or secondary to the surgical procedures throughout the continuum of care after the trauma (e.g., fasciotomy and debridement secondary to compartment syndrome). VML is a particularly troubling injury paradigm because mammalian skeletal muscle is not capable of extensive de novo muscle fiber regeneration after VML injury [Mase et al., 2010; Corona et al., 2013a, b]. As such, a VML injury consists of extensive fibrosis within the injured muscle and amongst surrounding tissues. Loss of limb function secondary to VML injury is therefore the result of reductions in muscle strength, joint range of motion, and other extensive musculoskeletal comorbidities [Garg et al., 2015].

The surgical and rehabilitative care for patients with extensive extremity trauma has primarily been pioneered within the US Military Health System, and significant advances in care have been achieved over the past decade of war. Specifically, the Military Treatment Facilities (Walter Reed National Military Medical Center, San Antonio Military Medical Center, and Naval Medical Center San Diego) and the three Advanced Rehabilitation Centers within these (Military Advanced Training Center, the Center for the Intrepid, and the Comprehensive Combat and Complex Casualty Care Program, respectively) have led the way in advancing the standard of care for patients with severely traumatized salvaged limbs. The utilization of a team approach, i.e., integration of advanced orthopedic surgical care combined with comprehensive physical rehabilitation programs and state-of-the-art technologies within these institutions, has produced salient advancements in returning injured servicemen and veterans to the highest level of function and quality of life.

Traditional rehabilitation therapies, both physical and/ or occupational therapy, intend to restore range of motion and increase muscle strength; they are the current clinical standard of care for limb salvage patients. While there are guidelines for physical therapy of muscle injuries of different etiology [Jarvinen et al., 2007], no such guidelines exist for VML-injured muscles and limbs, especially for patients who have sustained multiple severe concomitant injuries.
Clinical observations and the existing literature indicate that traditional physical therapy does not significantly restore muscle strength or limb function in patients with severe limb trauma that involves VML [Mase et al., 2010; Gentile et al., 2014; Sicari et al., 2014; Garg et al., 2015]. This lack of efficacy of traditional physical therapy paradigms in promoting functional recovery after VML is likely the result of the polytraumatic nature of the injuries (e.g., concomitant bone fracture, altered neural recruitment, tethering, and contracture) and attenuated regenerative potential of the remaining muscle mass. To address this clinical unmet need, innovative approaches such as the utilization of orthotic devices [Bedigrew et al., 2014] have been developed and utilized to provide functional improvements in the salvaged limbs although significant improvements in muscle strength per se remain low.

The capacity to improve the strength of VML-injured muscle via rehabilitation is dependent on: (1) the number of muscle fibers remaining, (2) the ability to activate the survived fibers, and (3) the capacity of the survived fibers to hypertrophy (i.e., increase in force-generating sarcomeric proteins per fiber) or potential promotion of fiber hyperplasia (i.e., increase in the total number of fibers per muscle). While rehabilitation of the VML-injured muscle is capable of promoting limited muscle fiber hypertrophy, it is unlikely to promote extensive fiber hyperplasia of a significant magnitude to replace the ample lost muscle fibers due to VML. Therefore, as the severity of VML injury increases, achievement of maximal restoration of muscle will require de novo regeneration of lost muscle fibers. As such, VML presents an ideal indication for innovative regenerative medicine therapies intended to promote de novo muscle fiber regeneration and improve skeletal muscle strength. Regenerative therapies definitive for VML are currently not part of the standard of care in military or civilian medicine, though emerging therapies are in various stages of development or clinical testing. By and large, the regenerative medicine field has made significant advancements in developing a broad spectrum of short- and long-term solutions for VML, and multiple approaches have demonstrated potential to improve muscle strength with varying degrees of successful muscle fiber regeneration.

Looking forward to the requirements for true clinical success in reestablishing function to traumatized limbs with VML injuries, the need to establish combinatorial rehabilitative and regenerative therapies is imminent. The benefits of combined physical rehabilitation and regenerative medicine are likely to enhance independent treatment efficacy, especially if each is prescribed appro- 
priately and with an individualized, patient-specific approach. To that end, the science of combined therapeutic approaches to regenerative rehabilitation [Perez-Terzic and Childers, 2014] is still in its infancy. At this point, numerous questions exist regarding patient-specific physical rehabilitation regimens and regenerative therapies. The focus of this review is to highlight current clinical strategies of rehabilitation in patients with VML, present generalizable findings of preclinical regenerative and rehabilitative investigations of VML, and put forth evidence-based opportunities for multidisciplinary collaboration between these clinical and research fields.

\section{Clinical Perspective on Rehabilitation and Regeneration following VML}

\section{Characterization of VML Injury}

Etiology

The primary etiology of VML injuries involves an abrupt frank loss of muscle fibers in otherwise healthy muscle (trauma) or previously injured muscle that requires evacuation or debridement. Therefore, VML injury is distinct from other less destructive forms of muscle injury (e.g., strain and snakebite) that leave remnants of the musculature in situ. The relative incidence of VML in orthopedic trauma is currently unknown. However, observational studies and anecdotal reports indicate VML is a pervasive problem among high-energy orthopedic injuries. In civilian limb extremity trauma patients, prospective observational analysis found that approximately half of patients presented either 'considerable muscle injury to 2 or more groups or loss of 1 entire group' or 'compartment or crush syndrome', which is predicted to produce VML following evacuation [Bosse et al., 2002]. Moreover, these soft tissue injuries resulted in severe disability 2 years after injury (sickness impact profile $>10$ ). Among battlefield orthopedic injuries in recent conflicts, over half are specifically to the soft tissue and an additional quarter are open fractures, which involve comorbid severe muscle tissue injury [Owens et al., 2007; Cross et al., 2011]. A recent retrospective analysis indicated that in an open tibia fracture cohort, VML injury accounted for the majority $(65 \%)$ of a patient's disability [Corona et al., 2015].

\section{Natural History}

The natural history of VML remains mostly undefined. A consistent observation among definitive VML clinical studies is the irrecoverable reduction and loss of limb function [Mase et al., 2010; Gentile et al., 2014; Sicari et al., 2014; Garg et al., 2015]. Functional limb deficits appear to be primarily due to a restricted range of motion and loss of muscle strength, i.e., reductions in isometric or isokinetic force or torque that manifest diminution of work and power [Corona et al., 2013a; Garg et al., 2015]. Wound exudates after severe trauma that are likely to present a loss of muscle tissue and require debridement (an injury pattern consistent with VML) have elevated concentrations of inflammatory cytokines and chemokines (IL-1 $\beta$, IL- 6 , and MCP-1) at prolonged time points after injury [Forsberg et al., 2014]. Based on these findings, VML injuries appear to present an abrupt irrecoverable frank loss of skeletal muscle tissue that incites a heightened and prolonged inflammatory response, which eventually causes extensive compartmental fibrosis. The frank loss of tissue also likely presents heterogeneous architectural changes in the remaining muscle, although this is not yet completely characterized [Garg et al., 2015]. Animal models predict that after the initial traumatic loss of muscle tissue, repeated phases of degeneration, repair, and remodeling ensue for an extended period after injury [Corona et al., 2013a, b], raising concern that the regenerative potential of the remaining muscle may become diminished in a manner similar to muscular dystrophies and/or nondystrophic myopathies. In support of this possibility, a prospective study of patients with severe limb extremity trauma demonstrated worsening sickness impact profile scores during the initial 7 years after injury [MacKenzie et al., 2005]. In addition, a retrospective study of physical assessment board evaluations of a cohort of wounded service members with type III open fracture found a progressive worsening of VMLrelated disability ratings [Rivera and Corona, 2016]. Furthermore, due to the nature of these injuries especially among the battlefield injured, many VML injuries occur relatively early in adulthood, thus long-term degeneration of the muscle tissue may contribute to the development and/or acceleration of other comorbidities (e.g., low back pain, osteoarthritis, metabolic syndrome, and cardiovascular disease).

\section{Rehabilitation following VML}

Currently, acute VML injuries are not definitively treated with functionally restorative regenerative therapies or surgical procedures. For instance, in open fracture cases, type IIIa fractures receive no treatment of VML adjacent to traumatized bone and type IIIb may receive a muscle or fasciocutaneous flap with the clinical intent to cover and support bone healing, that is salvage of a likely 
dysfunctional limb. Therefore, the impact that VML has on local and systemic wound healing and long-term disability is considered part of the natural sequelae of orthopedic trauma. In this context, physical rehabilitation is currently the only direct therapy for VML injuries. Specific to defined VML injuries, physical therapy does not appear to promote clinically appreciable or meaningful improvements in limb function, muscle strength, or joint range of motion [Mase et al., 2010; Gentile et al., 2014; Sicari et al., 2014; Garg et al., 2015]. Given the small number of reports, it is not possible to determine why the traumatized musculature does not respond to physical therapy. While the loss of contractile tissue is innate to VML etiology, the proliferative fibrotic response from the injury may also significantly impair limb function. Additional considerations to the lack of response to physical therapy alone could be due to comorbid conditions and the dysfunctional ability of the muscle to respond to exercise. Future studies should be designed to examine gene regulation, inflammatory responses, metabolic profiles, and exercise responsiveness as a consequence of VML injury both initially and years following injury in efforts to more appropriately design therapeutic options.

Nontraditional rehabilitation paradigms may be necessary to successfully rehabilitate severely traumatized limbs. As an example, improved function in limb salvage patients may be achieved in a comprehensive rehabilitation program that incorporates an energy-returning orthosis, which is designed to mitigate lower extremity disability and allow involvement in occupational and sport activities. The inclusion of an energy-returning orthosis with a physical therapy program improved performance towards healthy control values on a standardized battery of functional assessments (e.g., timed stair ascent) and self-reported functional outcomes, and has aided in the return to active duty in a small cohort of patients. [Patzkowski et al., 2012; Bedigrew et al., 2014; Blair et al., 2014]. These findings broadly suggest efficacy of physical rehabilitation for severely traumatized limbs. However, it is important to note that the effect of orthosis-assisted rehabilitation programs on muscle or limb function, once the orthosis is removed, has not been reported. Moreover, randomized prospective multicenter clinical studies are required to determine the broader therapeutic benefit of energy-returning orthoses.

\section{Combined Rehabilitation and Regeneration Therapy}

To date, only three clinical case reports have been published in which VML injuries were definitively treated with a regenerative medicine therapy (i.e., decellularized extracellular matrix, ECM) [Mase et al., 2010; Gentile et al., 2014; Sicari et al., 2014]. In these reports, all patients underwent traditional physical therapy before and after surgical implantation of the ECM. In each case, physical rehabilitation alone did not appear to improve functional outcomes. The first application of an ECM was performed in a Marine who was wounded by an improvised explosive device blast and presented chronic quadriceps VML secondary to femur open fracture [Mase et al., 2010]. The soft tissue loss following open fracture was treated with a latissimus dorsi muscle flap and the limb was successfully salvaged. Approximately 3.5 years after the injury, the patient presented with a $72 \%$ loss of isokinetic knee extensor strength despite having undergone traditional physical therapy for $>1.5$ years. The ECM implantation and resumed physical therapy program consisting of loading exercise directed at reestablishing function about the knee resulted in a $13 \%$ improvement in knee extensor strength 4 months after injury, assessed using a Biodex isokinetic dynamometer. However, a $68 \%$ strength deficit was retained in comparison to preoperative contralateral control values. An ongoing clinical trial reported 5 cases in whom an ECM was implanted at the site of VML and an injury-specific physical therapy regimen was performed [Sicari et al., 2014]. The VML injuries were localized within the quadriceps or anterior compartment of the lower limb and estimated as an $~ 60-90 \%$ loss of muscle tissue. Isometric muscle strength measured via a handheld dynamometer improved in 3 patients (range, $\sim 20-136 \%$ ) and did not change in the remaining 2 patients. Values before injury are not reported, disallowing assessment of the residual strength deficit. Lastly, a follow-on case report to Sicari et al. [2014] described a physical therapy regimen prescribed to an individual patient treated with ECM for quadriceps VML [Gentile et al., 2014]. In this patient, isometric knee extensor strength increased by $20 \%$ from before to 27 weeks after surgical implantation of the ECM. Compared to contralateral knee extensor strength, the ipsilateral limb presented an $89 \%$ deficit before surgery and an $87 \%$ deficit 27 weeks after ECM implantation and physical therapy.

\section{Future Directions}

The overarching goal is to develop and prescribe individualized, patient-specific physical rehabilitation and regenerative medicine therapies capable of providing the optimal functional performance and quality of life for patients with VML. However, before this can be achieved, numerous aspects of VML injuries require further investigation from basic characterization to chronic patho- 
physiological problems. A prospective analysis of VML injuries is needed to determine important therapeutic boundary conditions: such as to (1) determine what size VML per muscle or muscle unit results in loss of limb function or quality of life; (2) elucidate discrete mechanisms of functional loss (i.e., loss of muscle tissue vs. disabling fibrosis); (3) characterize the natural history of VML injuries along with other comorbid conditions, and (4) perform biological analyses of the traumatized muscle to determine the histological, cellular, and molecular environment with which regenerative medicine and rehabilitation interact mechanistically. Furthermore, prospective studies investigating optimal strategies to rehabilitate VML-injured muscles at discrete time points after injury are also needed.

Given the apparent modest unresponsiveness of clinical VML-injured muscle to traditional physical therapy, nontraditional methods may need to be explored, with consideration of the potential adverse effects of overloadinduced secondary muscle injury and time since initial injury. Clearly, regenerative therapies that promote de novo regeneration of the lost muscle tissue are needed to address this complex clinical problem. While clinical solutions to address the need for ongoing functional gains following VML are necessary, preclinical modeling and investigations of regenerative rehabilitation for VML injuries are crucial to understand fundamental mechanisms related to strength gain and loss, and de novo regeneration, and test possible mechanistically driven therapeutic approaches following VML.

\section{Preclinical Perspective on Rehabilitation and Regeneration following VML}

\section{Physiology of Skeletal Muscle Strength Gains}

Major contributors to force loss following skeletal muscle injury include structural failure, loss of contractile proteins, and excitation-contraction coupling failure [Warren et al., 2001]. It therefore follows that measurable gains in muscle strength are possible through hypertrophy, hyperplasia, and/or augmented neural interactions. The use of animal models has clearly indicated that VML results in significant structural failure and extensive loss of contractile protein content, which is the primary target of current regenerative initiatives (i.e., regenerating the lost contractile tissue). At the core of any successful therapeutic physical rehabilitation paradigm is the plasticity of skeletal muscle function. Broadly defined, functional plasticity of skeletal muscle is classified as the ability of skeletal muscle to adapt to perceived mechanical or chemical cues in order to improve its functional capacity and efficiency. The adaptions that occur may lead to changes in the metabolic or contractile phenotype (i.e., increased strength) and are typically determined by the nature of stressors placed on the residing muscle fibers. Physical rehabilitation and regenerative medicine therapies may need to target the remaining muscle mass in order to promote long-term, sustainable gains in skeletal muscle strength following VML. Hypertrophy, hyperplasia, and neural interaction-related mechanisms of strength gains are discussed further in the ensuing sections.

\section{Hypertrophy}

First, hypertrophy is the increase in muscle mass or size due to individual myofibers increasing in size, thus increasing the overall cross-sectional area of the muscle and ultimately positively affecting muscle strength. Notably as myofiber hypertrophy there is an increase in all fiber components, such as the sarcoplasmic reticulum, ttubules, contractile protein content, and myonuclei, etc., and a net positive increase in protein balance. The increased size of individual myofibers results in significant strength gains which are notable with specific force measurements (i.e., force normalized for physiological crosssectional area). Hypertrophy has been examined using various preclinical models [see for review Lowe and Alway, 2002], such as compensatory synergistic ablation [Schiaffino and Hanzlikova, 1970; Gutmann et al., 1971; Ianuzzo and Chen, 1977], progressive resistance exercise via squat-like apparatus [Klitgaard, 1988; Tamaki et al., 1992] or ladder climbing [Hellyer et al., 2012], chronic stretching via casting in a lengthened position [Goldberg et al., 1975; Kelley, 1996], and electrical stimulation via chronically implanted electrodes [Walters et al., 1991]. Additional understanding of the modulation of growth factor- or hormone-related hypertrophy has yet to be examined in a VML model, but agents such as IGF-1 [Sandri et al., 2013] or myostatin antibodies [Lee and McPherron, 2001] could be possible interventions that have been known to induce hypertrophy. Notably, each model or intervention has strengths and weaknesses (specifics are outside the scope of this review), and the practicality and efficacy of initiating many of these following VML have yet to be determined.

\section{Hyperplasia}

Second, hyperplasia or the formation of new myofibers occurs minimally to produce increases in muscle strength. Currently, there is no support of any hyperpla- 
sia occurring following VML specifically within the defect area. Nevertheless, preclinical models can induce rapid strength gains through hyperplasia, specifically by chronic stretching induced by casting which can rapidly induce hyperplasia [Alway et al., 1990]. Combining strength-increasing modalities such as chronic stretching and electrical stimulation has been shown to be synergistic for strength gains related to hyperplasia [Goldspink et al., 1995]. Both clinical and preclinical applications of chronic stretch-induced hyperplasia need to be examined following VML, as de novo generation of myofibers following VML is necessary for functional recovery.

Satellite cells, the resident muscle stem cell progenitor, have a role in both of these processes, either by fusing to existing fibers or fusing together in the formation of new fibers during hypertrophy and hyperplasia, respectively. Any possible intervention regarding hypertrophy or hyperplasia should address the activation of satellite cells for subsequent gains in strength. Unfortunately, current examinations of rehabilitation following VML have not supported strength gains via hypertrophy or hyperplasia mechanisms [Aurora et al., 2014]. Notably, it is likely that optimal strength gains after VML will require intervention at the primary injury site and the remaining musculature. However, this alone should not diminish the importance of strength maintenance or possible improvements in the remaining musculature.

Following VML, there is likely disuse of remaining tissue for some time after injury. Disuse following bed rest [Koukourikos et al., 2014], hindlimb unloading/suspension [Warren et al., 2004; Wenke et al., 2010; Greising et al., 2011a], microgravity [Fitts et al., 2000; Trappe, 2009], limited physical activity [Novotny et al., 2013], and as a result of aging [Faulkner et al., 2007; Fielding et al., 2011] or degeneration [Pansarasa et al., 2014], all result in significant dysfunction, atrophy, and force loss of skeletal muscle through various mechanisms. In parallel to this, the remaining musculature following VML may also undergo secondary muscle injury due to mechanical overload, also resulting in significant muscle dysfunction to the remaining muscle, resulting in a phenotype similar to muscular dystrophies and/or nondystrophic myopathies. With this possible secondary muscle damage, any therapeutic options for VML must address significant force deficits at the injury site and any secondary deficits in the remaining musculature due to subsequent disuse, degeneration, and/or reoccurring injury. This may require targeted approaches for the improvement in muscle function at various injury sites and the surrounding tissue (e.g., bone or nerves). The use of preclinical models has a strong advantage in these types of studies. Unfortunately, it is difficult to discriminate between functional outcomes attributed to discrete portions of the traumatized musculature, i.e., defect vs. remaining musculature. Such limitations in methodology and technology need to be addressed using a multidisciplinary approach of physiologists, physicians, physical therapists, and biomedical engineers.

\section{Neural Interactions}

The final mechanism is neural interactions, which have a role in strength gains usually during the onset of a novel training or rehabilitation program. Primarily, the neural response occurs due to an optimization and synchronization of motor unit recruitment, efficiency in system activation, and deficits in neural inhibition [Moritani and deVries, 1979; Ploutz et al., 1994]. This neural response normally occurs prior to any hypertrophy or hyperplasia of the muscle. The current field examining VML has generally overlooked the resulting effects that intramuscular or peripheral nerve damage have on residual neuromuscular functional deficits. One could hypothesize that functional deficits could be due to disturbances at various levels of the neuromuscular system, and, in parallel, any expected neural interactions due to rehabilitation or regeneration following VML may be limited due to peripheral nerve injury.

Examinations of interactions between motoneurons and the muscle fibers they innervate (i.e., within a motor unit) are necessary to develop treatments to preserve and promote regeneration and repair of neuromuscular function following VML. Substantial evidence supports that the central nervous system influences the fiber type of muscles (i.e., myosin heavy chain isoform expression) wherein an individual motor unit innervates a group of muscle fibers of the same type and matched contractile and fatigue properties [for review, see Greising et al., 2012]. For example, under normal physiologic conditions, a slow (type $\mathrm{S}$ ) motor unit innervates muscle fibers that are classified as type I and express the slow myosin heavy chain isoform. All the muscle fibers innervated throughout an individual motor unit will share metabolic, contractile, and fatigue characteristics. Interactions between the motor unit and muscle fiber have yet to be examined following VML, but disruptions in trophic signaling are likely with the vast loss of muscle and probable nerve damage. It is possible that the loss of nerve- or muscle-derived trophic signaling following VML may result in frank motoneuron death, muscle fiber denervation, impaired fiber reinnervation, motor unit expansion, and 
clustering of muscle fiber types, all of which will limit the functional capacity of the muscle. Future studies are needed to examine these possibilities following VML. The understanding of neural interactions following VML may allow for the development of targeted interventions that exploit muscle plasticity through neural interaction. Furthermore, targeting trophic signaling of specific motor unit types, and as such myosin heavy chain types, could provide optimization of neural activation, promotion of spared neural pathways, and greater efficacy of regenerative and rehabilitative therapies.

\section{Physiological Improvement Threshold}

Maximal size and force gains possible following various preclinical functional models can range from a 10 to $50 \%$ increase in myofiber size (i.e., hypertrophy), 10 $100 \%$ increases in muscle mass, and $10-100 \%$ increases in overall strength [Lowe and Alway, 2002]. With this, one could hypothesize that following VML with just the immediate frank loss of skeletal muscle tissue, there may be a limit on the magnitude of strength gains that are physiologically possible, i.e., the amount of size and force gains that an individual skeletal muscle can undergo following traditional rehabilitation paradigms may be limited by the significance of the volume loss and the parallel strength loss. Therefore, any size and force gains that are necessary above this threshold require de novo regeneration of myofibers. Current research initiatives need to address this theoretical threshold of functional gains with the use of combined rehabilitation and regenerative medicine.

The ability to examine functional outcomes following VML has been improved with the development and standardization of various preclinical extremity injury models. In the rodent, VML models in the tibialis anterior (TA) [Wu, 2012; Corona et al., 2013a; Garg et al., 2015], gastrocnemius [Merritt et al., 2010; Aurora et al., 2015], quadriceps [Willett et al., 2013; Li et al., 2014], and latissimus dorsi [Chen and Walters, 2013] muscles have been developed to allow neuromuscular strength testing. Each of these models present chronic strength deficits. Importantly, the use of preclinical VML models enables specific examination of various rehabilitation and regenerative medicine paradigms that are otherwise not possible in traditional clinical trials. The use of preclinical animal models allows for an untreated VML group, be that no rehabilitation or regenerative therapy. With these models building on the current understanding, new experimental approaches and combined therapies need to be examined following VML. The ensuing preclinical syn- opsis will focus on the functional outcomes of the hindlimb muscles following rehabilitation and/or regenerative approaches.

\section{Rehabilitation following VML}

To date, there has only been limited investigation specifically examining skeletal muscle function following a rehabilitation paradigm designed to address skeletal muscle function following VML. Aurora et al. [2014] used voluntary wheel running in a rat TA muscle VML model designed to remove $\sim 20 \%$ of the muscle mass. Animals underwent functional testing after 2 or 8 weeks, and half were given access to running wheels 1 week after VML injury, respectively. Running distances were $\sim 1.7$ $\mathrm{km} /$ day for the duration of the 7-week study, resulting in a $17 \%$ increase in anterior crural muscle torque and a $13 \%$ increase in TA muscle mass; however, there was no promotion of hypertrophy or hyperplasia as determined by myofiber cross-sectional area or number, respectively. Furthermore, there was a significant increase in the number of centrally located nuclei in the myofibers, presenting a putative dystrophic-like phenotype in the muscle. Moreover, the loss of $\sim 20 \%$ of muscle mass results in a pseudo synergistic ablation model between the TA and extensor digitorum longus muscles. Unfortunately, wheel running did not result in a significant increase in the mass of the extensor digitorum longus muscle after VML, while this was found in the sedentary group. This report provided evidence that running after VML likely affected the ability of the muscle to transmit force while having no effect on hypertrophy (i.e., force production).

\section{Future Directions}

The use of physical rehabilitation following VML needs to focus on the modality, the time since injury for initiation, and the progression through long-term maintenance. Rehabilitation modalities that are currently examined to address strength loss (see above) should be examined in models of VML. Additionally, commonly used modalities such as wheel running [Warren et al., 2007; Landisch et al., 2008; Greising et al., 2011b; Walters et al., 2015] or treadmill running [Armstrong and Taylor, 1982], weighted wheel running [Ishihara et al., 1998; Konhilas et al., 2005; Legerlotz et al., 2008; Call et al., 2010], eccentric muscle training [Newham et al., 1987; Ingalls et al., 2004; Call et al., 2011], and range of motion exercises [Moriyama et al., 2013; Peixinho et al., 2014] could be used either in isolation or combination to improve functional recovery after VML. 
While wheel running did not result in functional gains in this recent report [Aurora et al., 2014], it is possible that alterations in the running paradigm may prove beneficial. Specifically, modifying running protocols to control the running distances and progressively increase weekly distances and work may limit any additional damage due to running. Other voluntary rehabilitation protocols could include the use of progressive resistance voluntary wheel running. This model that mimics voluntary progressive resistance training has been shown to result in hypertrophy, as indicated by increased myofiber cross-sectional area [Ishihara et al., 1998; Konhilas et al., 2005]. It is possible that beginning a rehabilitation protocol with limited unweighted wheels and progressing into weighted wheels could significantly improve the use of this voluntary modality to induce first increased ambulation and range of motion, then hypertrophy after VML.

\section{Regeneration following VML}

Regenerative therapies and tissue engineering strategies have aimed to improve muscle strength primarily by promoting de novo muscle fiber regeneration within a VML defect. Recognizing that the primary endogenous pillars of skeletal muscle regeneration are the satellite cell, basal lamina, and an appropriate inflammatory response, regenerative approaches have replaced or substituted components resident in the muscle that orchestrate the spatiotemporal events that underlie mammalian muscle fiber regeneration [Ciciliot and Schiaffino, 2010]. Current approaches have generally delivered a scaffold (native or decellularized ECM or hydrogel) with or without various cell types (satellite cells, mesenchymal stem cells, or muscle-derived cells), which may be delivered as an autologous muscle tissue derivative. Additionally, some approaches include ex vivo preconditioning stimuli or cell culture procedures. These approaches have been tested in an array of extremity VML models, and through these experiments significant discovery has been made regarding boundary conditions for de novo muscle fiber regeneration. The attributes of distinct regenerative approaches are described.

\section{Muscle Grafts}

The ability of skeletal muscle to regenerate is quite remarkable. Experiments performed over 50 years ago demonstrated that a muscle that was minced into small pieces $\left(\sim 1 \mathrm{~mm}^{3}\right)$ gradually regenerated a whole muscle with functional properties [Studitsky, 1964; Carlson, $1968,1970]$. During this pioneering era of muscle regeneration, Mauro [1961] discovered the satellite cell, and it was postulated that elements resident in muscles possessed regenerative properties. Modern-day Cre-recombinant technology has definitively demonstrated that skeletal muscle regeneration is absolutely dependent on the presence and activity of satellite cells (i.e., pax7 expression) [Lepper et al., 2011]. With this in mind, an autologous minced muscle graft procedure has been investigated for VML repair. Minced graft repair (prepared presurgically) of VML in a rat TA muscle has resulted in significant improvements in neuromuscular strength compared to nonrepaired [Corona et al., 2013a; Ward et al., 2015] and scaffold-repaired muscles [Garg et al., 2014]. The minced grafts promote appreciable de novo fiber regeneration, as indicated by the presence of myosin-positive fibers within the defect area [Corona et al., 2013a; Garg et al., 2014]. Additionally, de novo fiber regeneration promoted by minced grafts has been determined via counting all of the muscle fibers within uninjured and nonrepaired and minced graft-repaired muscles [Ward et al., 2015]; minced grafts regenerated $~ 63 \%$ of the lost fibers (3,242 fibers regenerated out of 5,118 fibers lost). A rat quadriceps VML has also been repaired with autologous muscle grafts (whole or minced) [Li et al., 2014]. Neither autograft (whole or minced) resulted in appreciable functional improvement of the skeletal muscle 2 or 4 weeks after injury. However, regeneration was apparent within the VML defect, with centrally located nuclei indicating regeneration of fibers. Furthermore, the minced muscle grafts had less fibrosis present than whole grafts or no-repair groups. Importantly, the fibers regenerated after minced graft repair result primarily from graft-delivered myogenic cells based on GFP donor tissue studies [Ward et al., 2015], and they become functionally innervated, as determined by glycogen depletion following repeated neural stimulation [Corona et al., 2013a]. However, the orientation and morphology of the regenerated fibers can be suboptimal, resulting in less strength recovery than is predicted by the amount of tissue regenerated [Ward et al., 2015]. This mismatch in the functional gains and the number of fibers regenerated highlights the yet undetermined aspects related to functional outcomes following regenerative therapies. Specifically, this emphasizes the need to understand the architectural changes of the skeletal muscle both following VML and treatment strategies. This understanding would address changes in the length-tension relationship of muscle and the transmission of force both laterally and longitudinally after regeneration. 
ECM Scaffolds

Historically, the purported role of ECM has been to simply provide mechanical support. However, it is now recognized that the ECM of an individual tissue or organ is comprised of a specific and unique biochemical composition and three-dimensional architecture which plays a central role in directing cell communication and function in situ. When surgically placed within a VML defect, ECM scaffolds serve as a bioactive matrix upon which site-appropriate tissue formation may occur subsequent to host cell infiltration, matrix degradation, and tissue remodeling. Current ECM scaffold technologies and configurations are numerous and technically diverse [for review, see Wolf et al., 2015]. The most robust presentation of ECM-mediated muscle fiber regeneration was presented in a dog musculotendon injury model [Turner et al., 2010]. Extensive muscle fiber formation of similar morphology to native muscle tissue had regenerated by 6 months after injury. A more recent study using a similar injury model in rats observed that no regenerative events occurred in the space of implanted ECM, which was eventually resorbed [Aurora et al., 2015]. The discrepant findings between studies cannot be determined, as numerous methodological differences exist, to include the source and content of the ECM. For example, ECM repair of a mouse thigh VML model using urinary bladder matrix (UBM) has been reported to promote the formation of a likely subphysiological number of muscle fibers primarily in close proximity to the adjacent, remaining musculature [Sicari et al., 2014]. Similar results have been reported in TA and latissimus dorsi VML models following implantation of a commercial version of UBM or a custom-made skeletal muscle ECM or UBM, wherein, despite little evidence of de novo fiber regeneration, neuromuscular strength improvements were observed [Chen and Walters, 2013; Corona et al., 2013b, 2014; Aurora et al., 2015]. In each study, ECMmediated deposition of fibrous tissue in the defect is purported to improve the efficiency of force transmission from the remaining musculature. Of note, the use of ECM scaffolds and similar technologies (collagen hydrogel) in a VML defect does not always promote neuromuscular strength improvements [Merritt et al., 2010; Ward et al., 2015], indicating the potential for divergent material composition requirements per injury condition to improve force transmission after VML. Based on observations that putative alternative myogenic stem cells derived from the vasculature infiltrate implanted ECMs [Turner et al., 2010; Garg et al., 2014; Sicari et al., 2014; Aurora et al., 2015], while satellite cells are largely absent
[Garg et al., 2014; Aurora et al., 2015], restricted migration of satellite cells from the remaining musculature appears to be a primary limitation to ECM-mediated de novo regeneration. While ideally these approaches promote de novo regeneration of fibers that incorporate in the remaining skeletal muscle tissue, it is important to highlight that the ultimate goal of these techniques is to address functional deficits of skeletal muscle, and therefore therapies with low regenerative potential which demonstrate safety and efficacy in increasing strength are invaluable for the treatment of heterogeneous VML injuries.

\section{Tissue-Engineered Muscle Repair Constructs}

Tissue-engineered muscle repair (TEMR) constructs can be broadly defined as any technology which aims to combine scaffold material with one or more of the various cellular components (e.g., stem/progenitor cells). Ideally, the scaffold material utilized within a TEMR construct would provide an appropriate microenvironmental niche capable of promoting the proliferation, differentiation, and/or maturation of myogenic stem/progenitor cells. To this end, numerous materials have shown to improve the myogenic response of myoblasts when compared to standard culture conditions. Additionally, TEMR constructs can be generated with or without the addition of ex vivo mechanical preconditioning. In recent years, numerous studies have been conducted in which various TEMR constructs, differing in composition (e.g., skeletal muscle ECM, collagen I, or hyaluronic acid), cellular components (e.g., myoblasts or bone marrow-derived mononuclear cells), and preconditioning strategies (e.g., cyclic mechanical strain), have been evaluated in several different VML models, each eliciting varying degrees of apparent host-mediated myogenesis, vascularization, and/or improvements in tissue function [Kroehne et al., 2008; Machingal et al., 2011; Rossi et al., 2011; Corona et al., 2014]. While these results are promising and provide support for continued investigation, FDA approval will have to be obtained before these technologies can be translated into the clinic.

\section{Future Directions}

These studies indicate significant promise for the regenerative repair of VML through continued optimization of current approaches and innovation in application. A range of magnitude (moderate to none) of fiber regeneration has been presented following regenerative treatment approaches in fairly simple VML models, which raises the question as to what a reasonable level of 
expectation is regarding de novo regeneration. This is especially highlighted by the fact that preclinical animal studies repair freshly injured VML defects devoid of pathological manifestations of chronic VML, as is currently encountered in the clinic. Ongoing research should be directed toward both immediate and delayed repair from the initial time of VML and examine regenerative repair strategies that span multiple muscles. No therapeutic strategy tested to date is without limitation or merit, and it remains important that multiple repair and regenerative tools are developed for the heterogeneous presentation of VML injuries among trauma patients.

\section{Combined Rehabilitation and Regeneration Therapy}

It is reasonable to hypothesize that combined therapies using rehabilitation and regenerative medicine paradigms would result in a synergistic effect on skeletal muscle function following VML. To date, limited research has addressed this important question. Corona et al. [2013a] examined the effect of activity on neuromuscular strength improvements in a rat TA muscle VML injury repaired with autologous minced grafts. Rats were either allowed normal cage activity or given access to running wheels for 7 weeks starting 1 week after injury. The rats ran $\sim 1.8 \mathrm{~km} /$ day over 7 weeks (initiated 1 week after injury), which resulted in a significant increase $(\sim 100 \%)$ in the recovery of TA muscle strength that was observed with sedentary minced graft repair, suggesting synergy between regenerative and rehabilitative therapies. In the same rat TA muscle VML injury model with similar experimental design (i.e., normal cage activity vs. wheel running for 7 weeks beginning either 1 or 4 weeks after injury), the synergy between rehabilitation and ECM repair was recently investigated [Aurora et al., 2015]. As noted previously, the ECM repair promoted a significant improvement in isometric strength 8 but not 16 weeks after injury. However, voluntary wheel running did not further improve recovery of muscle strength regardless of the time physical rehabilitation was initiated. In consideration of the limitations of these studies, the findings suggest that physical rehabilitation amplifies strength improvements promoted by regenerative therapies only when significant de novo regeneration is achieved.

\section{Future Directions}

The use of combined therapies to approach therapeutic interventions needs to address VML using regenerative rehabilitation [Perez-Terzic and Childers, 2014]. For functionally significant hypertrophy and de novo fiber regeneration to occur, combined therapies are necessary. At present, the only rehabilitation therapy that has been investigated in preclinical animal models is voluntary wheel running. Additional modalities need to be explored to address this significant problem. For instance, therapies that involve both range of motion exercises and chronic electrical stimulation may promote both neural additions and hyperplasia following VML. Additionally, regenerative rehabilitation research needs to be addressed using a multifactorial approach that considers the state of the muscle throughout time following VML, not just the muscle immediately after injury.

\section{Closing Perspective on VML}

As a relatively young and growing field, significant advancements in both rehabilitation and regeneration following VML have been made over the past 10 years, as the incidence of VML injuries has increased extensively due to the recent military conflicts. Nonetheless, there are still gaps in the current understanding of the clinical pathophysiology of VML in the long term, and with this it is difficult to determine optimal therapeutic rehabilitation paradigms. Furthermore, the nature of VML injuries is highly heterogeneous, and individualized treatment plans likely need to be developed.

Both clinical and a range of preclinical research (i.e., small to large animal models) needs to address changes throughout the neuromuscular system and develop specific modalities and functional tests to quantify the efficacy of therapeutic interventions. While this review focused on the skeletal muscle, neighboring and supporting tissues need to be taken into consideration, such as bone, nerve, and vasculature; furthermore, interventions that focus on multi-limb and site repair are needed. Moreover, in healthy lean individuals, skeletal muscle makes up approximately half of body weight. Significant losses in muscle volume following VML likely impact the whole body metabolism, influencing comorbid diseases, including diabetes, obesity, and the metabolic syndrome, which is currently being overlooked. The examination of skeletal muscle also needs to be tackled using different approaches to understand the organization and architectural changes of skeletal muscle fibers following VML and various regenerative rehabilitation interventions. As the regenerative rehabilitation field continues to grow, a multidisciplinary and concerted effort amongst those examining and treating VML needs to be made. Future directions regarding regenerative rehabilitation 
following VML need to be addressed by multidisciplinary groups that examine the encompassing physiology, pathology, and specific needs of this patient population.

\section{Acknowledgments}

The authors acknowledge their funding support from the US Army Medical Research and Materiel Command, Clinical and Rehabilitative Medicine Research Program [Regenerative Medicine
(C_003_2015_USAISR) to B.T.C. and Neuromusculoskeletal Injury Rehabilitation (MR140099) to B.T.C.], the National Institute of Health (1R03EB018889-01A1 to C.L.D.), and the DoD-VA Extremity Trauma and Amputation Center of Excellence (Public Law 110-417, National Defense Authorization Act 2009, Section 723, to C.L.D.).

\section{Disclosure Statement}

The authors declare no conflict of interest.

\section{References}

Alway, S.E., W.J. Gonyea, M.E. Davis (1990) Muscle fiber formation and fiber hypertrophy during the onset of stretch-overload. Am J Physiol 259: C92-C102.

-Armstrong, R.B., C.R. Taylor (1982) Relationship between muscle force and muscle area showing glycogen loss during locomotion. J Exp Biol 97: 411-420.

Aurora, A., K. Garg, B.T. Corona, T.J. Walters (2014) Physical rehabilitation improves muscle function following volumetric muscle loss injury. BMC Sports Sci Med Rehabil 6: 41.

-Aurora, A., J.L. Roe, B.T. Corona, T.J. Walters (2015) An acellular biologic scaffold does not regenerate appreciable de novo muscle tissue in rat models of volumetric muscle loss injury. Biomaterials 67: 393-407.

Bedigrew, K.M., J.C. Patzkowski, J.M. Wilken, J.G. Owens, R.V. Blanck, D.J. Stinner, K.L. Kirk, J.R. Hsu; Skeletal Trauma Research Consortium (STReC) (2014) Can an integrated orthotic and rehabilitation program decrease pain and improve function after lower extremity trauma? Clin Orthop Relat Res 472: 3017-3025.

Blair, J.A., J.C. Patzkowski, R.V. Blanck, J.G. Owens, J.R. Hsu; Skeletal Trauma Research Consortium (STReC) (2014) Return to duty after integrated orthotic and rehabilitation initiative. J Orthop Trauma 28: e70-e74.

Bosse, M.J., E.J. MacKenzie, J.F. Kellam, A.R. Burgess, L.X. Webb, M.F. Swiontkowski, R.W. Sanders, A.L. Jones, M.P. McAndrew, B.M. Patterson, M.L. McCarthy, T.G. Travison, R.C. Castillo (2002) An analysis of outcomes of reconstruction or amputation after legthreatening injuries. N Engl J Med 347: $1924-$ 1931.

Call, J.A., M.D. Eckhoff, K.A. Baltgalvis, G.L. Warren, D.A. Lowe (2011) Adaptive strength gains in dystrophic muscle exposed to repeated bouts of eccentric contraction. J Appl Physiol 111: 1768-1777.

Call, J.A., J.N. McKeehen, S.A. Novotny, D.A. Lowe (2010) Progressive resistance voluntary wheel running in the $\mathrm{mdx}$ mouse. Muscle Nerve 42: 871-880.
Carlson, B.M. (1968) Regeneration of the completely excised gastrocnemius muscle in the frog and rat from minced muscle fragments. J Morphol 125: 447-472.

Carlson, B.M. (1970) Regeneration of the rat gastrocnemius muscle from sibling and non-sibling muscle fragments. Am J Anat 128: 21-31.

Chen, X.K., T.J. Walters (2013) Muscle-derived decellularised extracellular matrix improves functional recovery in a rat latissimus dorsi muscle defect model. J Plast Reconstr Aesthet Surg 66: 1750-1758.

Ciciliot, S., S. Schiaffino (2010) Regeneration of mammalian skeletal muscle. Basic mechanisms and clinical implications. Curr Pharm Des 16: 906-914.

Corona, B.T., K. Garg, C.L. Ward, J.S. McDaniel, T.J. Walters, C.R. Rathbone (2013a) Autologous minced muscle grafts: a tissue engineering therapy for the volumetric loss of skeletal muscle. Am J Physiol Cell Physiol 305: C761C775.

Corona, B.T., J.C. Rivera, J.C. Owens, J.C. Wenke, C.R. Rathbone (2015) Volumetric muscle loss leads to permanent disability following extremity trauma. J Rehabil Res Dev 52: 785792.

Corona, B.T., C.L. Ward, H.B. Baker, T.J. Walters, G.J. Christ (2014) Implantation of in vitro tissue engineered muscle repair constructs and bladder acellular matrices partially restore in vivo skeletal muscle function in a rat model of volumetric muscle loss injury. Tissue Eng Part A 20: 705-715.

Corona, B.T., X. Wu, C.L. Ward, J.S. McDaniel, C.R. Rathbone, T.J. Walters (2013b) The promotion of a functional fibrosis in skeletal muscle with volumetric muscle loss injury following the transplantation of muscle-ECM. Biomaterials 34: 3324-3335.

Cross, J.D., J.R. Ficke, J.R. Hsu, B.D. Masini, J.C. Wenke (2011) Battlefield orthopaedic injuries cause the majority of long-term disabilities. J Am Acad Orthop Surg 19(suppl 1): S1-S7.

Faulkner, J.A., L.M. Larkin, D.R. Claflin, S.V. Brooks (2007) Age-related changes in the structure and function of skeletal muscles. Clin Exp Pharmacol Physiol 34: 1091-1096.
Fielding, R.A., B. Vellas, W.J. Evans, S. Bhasin, J.E. Morley, A.B. Newman, G. Abellan van Kan, S. Andrieu, J. Bauer, D. Breuille, T. Cederholm, J. Chandler, C. De Meynard, L. Donini, T. Harris, A. Kannt, F. Keime Guibert, G. Onder, D. Papanicolaou, Y. Rolland, D. Rooks, C. Sieber, E. Souhami, S. Verlaan, M. Zamboni (2011) Sarcopenia: an undiagnosed condition in older adults. Current consensus definition: prevalence, etiology, and consequences. International Working Group on Sarcopenia. J Am Med Dir Assoc 12: 249-256.

Fitts, R.H., D.R. Riley, J.J. Widrick (2000) Physiology of a microgravity environment. Invited review: microgravity and skeletal muscle. J Appl Physiol (1985) 89: 823-839.

Forsberg, J.A., B.K. Potter, E.M. Polfer, S.D. Safford, E.A. Elster (2014) Do inflammatory markers portend heterotopic ossification and wound failure in combat wounds? Clin Orthop Relat Res 472: 2845-2854.

Garg, K., C.L. Ward, B.J. Hurtgen, J.M. Wilken, D.J. Stinner, J.C. Wenke, J.G. Owens, B.T. Corona (2015) Volumetric muscle loss: persistent functional deficits beyond frank loss of tissue. J Orthop Res 33: 40-46.

Garg, K., C.L. Ward, C.R. Rathbone, B.T. Corona (2014) Transplantation of devitalized muscle scaffolds is insufficient for appreciable de novo muscle fiber regeneration after volumetric muscle loss injury. Cell Tissue Res 358: 857-873.

Gentile, N.E., K.M. Stearns, E.H. Brown, J.P. Rubin, M.L. Boninger, C.L. Dearth, F. Ambrosio, S.F. Badylak (2014) Targeted rehabilitation after extracellular matrix scaffold transplantation for the treatment of volumetric muscle loss. Am J Phys Med Rehabil 93(11 suppl 3): S79-S87.

Goldberg, A.L., J.D. Etlinger, D.F. Goldspink, C. Jablecki (1975) Mechanism of work-induced hypertrophy of skeletal muscle. Med Sci Sports 7: 185-198.

Goldspink, D.F., V.M. Cox, S.K. Smith, L.A. Eaves, N.J. Osbaldeston, D.M. Lee, D. Mantle (1995) Muscle growth in response to mechanical stimuli. Am J Physiol 268: E288-E297. 
Greising, S.M., K.A. Baltgalvis, A.M. Kosir, A.L. Lee, S.J., A.C. McPherron (2001) Regulation of Moran, G.L. Warren, D.A. Lowe (2011a) Estradiol's beneficial effect on murine muscle function is independent of muscle activity. J Appl Physiol (1985) 110: 109-115.

-Greising, S.M., R.S. Carey, J.E. Blackford, L.E. Dalton, A.M. Kosir, D.A. Lowe (2011b) Estradiol treatment, physical activity, and muscle function in ovarian-senescent mice. Exp Gerontol 46: 685-693.

Greising, S.M., H.M. Gransee, C.B. Mantilla, G.C. Sieck (2012) Systems biology of skeletal muscle: fiber type as an organizing principle. Wiley Interdiscip Rev Syst Biol Med 4: 457-473.

Gutmann, E., S. Schiaffino, V. Hanzlikova (1971) Mechanism of compensatory hypertrophy in skeletal muscle of the rat. Exp Neurol 31: 451464.

-Hellyer, N.J., J.J. Nokleby, B.M. Thicke, W.Z. Zhan, G.C. Sieck, C.B. Mantilla (2012) Reduced ribosomal protein $\mathrm{s} 6$ phosphorylation after progressive resistance exercise in growing adolescent rats. J Strength Cond Res 26 1657-1666.

Ianuzzo, C.D., V. Chen (1977) Compensatory hypertrophy of skeletal muscle: contractile characteristics. Physiol Teach 6: 4-7.

Ingalls, C.P., J.C. Wenke, T. Nofal, R.B. Armstrong (2004) Adaptation to lengthening contraction-induced injury in mouse muscle. J Appl Physiol 97: 1067-1076.

-Ishihara, A., R.R. Roy, Y. Ohira, Y. Ibata, V.R. Edgerton (1998) Hypertrophy of rat plantaris muscle fibers after voluntary running with increasing loads. J Appl Physiol (1985) 84: 2183-2189.

-Jarvinen, T.A., T.L. Jarvinen, M. Kaariainen, V. Aarimaa, S. Vaittinen, H. Kalimo, M. Jarvinen (2007) Muscle injuries: optimising recovery. Best Pract Res Clin Rheumatol 21: 317-331.

Kelley, G. (1996) Mechanical overload and skeletal muscle fiber hyperplasia: a meta-analysis. J Appl Physiol (1985) 81: 1584-1588.

Klitgaard, H. (1988) A model for quantitative strength training of hindlimb muscles of the rat. J Appl Physiol (1985) 64: 1740-1745.

Konhilas, J.P., U. Widegren, D.L. Allen, A.C. Paul, A. Cleary, L.A. Leinwand (2005) Loaded wheel running and muscle adaptation in the mouse. Am J Physiol Heart Circ Physiol 289: H455-H465.

Koukourikos, K., A. Tsaloglidou, L. Kourkouta (2014) Muscle atrophy in intensive care unit patients. Acta Inform Med 22: 406-410.

-Kroehne, V., I. Heschel, F. Schugner, D. Lasrich, J.W. Bartsch, H. Jockusch (2008) Use of a novel collagen matrix with oriented pore structure for muscle cell differentiation in cell culture and in grafts. J Cell Mol Med 12: 16401648 .

Landisch, R.M., A.M. Kosir, S.A. Nelson, K.A. Baltgalvis, D.A. Lowe (2008) Adaptive and nonadaptive responses to voluntary wheel running by mdx mice. Muscle Nerve 38 : 1290-1303. myostatin activity and muscle growth. Proc Natl Acad Sci USA 98: 9306-9311.

Legerlotz, K., B. Elliott, B. Guillemin, H.K. Smith (2008) Voluntary resistance running wheel activity pattern and skeletal muscle growth in rats. Exp Physiol 93: 754-762.

Lepper, C., T.A. Partridge, C.M. Fan (2011) An absolute requirement for Pax7-positive satellite cells in acute injury-induced skeletal muscle re-

Li, M.T., N.J. Willett, B.A. Uhrig, R.E. Guldberg, G.L. Warren (2014) Functional analysis of limb recovery following autograft treatment of volumetric muscle loss in the quadriceps femoris. J Biomech 47: 2013-2021.

Lowe, D.A., S.E. Alway (2002) Animal models for inducing muscle hypertrophy: are they relevant for clinical applications in humans? J Orthop Sports Phys Ther 32: 36-43.

Machingal, M.A., B.T. Corona, T.J. Walters, V. Kesireddy, C.N. Koval, A. Dannahower, W. Zhao, J.J. Yoo, G.J. Christ (2011) A tissue-entional restoration of an irrecoverable muscle injury in a murine model. Tissue Eng Part A 17: 2291-2303.

MacKenzie, E.J., M.J. Bosse, A.N. Pollak, L.X. Webb, M.F. Swiontkowski, J.F. Kellam, D.G. Smith, R.W. Sanders, A.L. Jones, A.J. Starr, M.P. McAndrew, B.M. Patterson, A.R. Burgess, R.C. Castillo (2005) Long-term persis tence of disability following severe lowerlimb trauma. Results of a seven-year followup. J Bone Joint Surg Am 87: 1801-1809.

Mase, V.J., Jr., J.R. Hsu, S.E. Wolf, J.C. Wenke, D.G. Baer, J. Owens, S.F. Badylak, T.J. Walters (2010) Clinical application of an acellular biologic scaffold for surgical repair of a large, traumatic quadriceps femoris muscle defect. Orthopedics 33: 511.

Mauro, A. (1961) Satellite cell of skeletal muscle fibers. J Biophys Biochem Cytol 9: 493-495.

Merritt, E.K., D.W. Hammers, M. Tierney, L.J. Suggs, T.J. Walters, R.P. Farrar (2010) Functional assessment of skeletal muscle regeneration utilizing homologous extracellular matrix as scaffolding. Tissue Eng Part A 16: 1395-1405.

Moritani, T., H.A. deVries (1979) Neural factors versus hypertrophy in the time course of muscle strength gain. Am J Phys Med 58: 115-130.

Moriyama, H., Y. Tobimatsu, J. Ozawa, N. Kito, R. Tanaka (2013) Amount of torque and duration of stretching affects correction of knee contracture in a rat model of spinal cord injury. Clin Orthop Relat Res 471: 3626-3636.

Newham, D.J., D.A. Jones, P.M. Clarkson (1987) Repeated high-force eccentric exercise: effects on muscle pain and damage. J Appl Physiol

Novotny, S.A., M.D. Eckhoff, B.C. Eby, J.A. Call, D. Nuckley, D.A. Lowe (2013) Musculoskeletal response of dystrophic mice to short term, low intensity, high frequency vibration. J Musculoskelet Neuronal Interact 13: 418429 . generation. Development 138: 3639-3646. gineered muscle repair construct for func(1985) 63: 1381-1386.
Owens, B.D., J.F. Kragh, Jr., J. Macaitis, S.J. Svoboda, J.C. Wenke (2007) Characterization of extremity wounds in Operation Iraqi Freedom and Operation Enduring Freedom. J Orthop Trauma 21: 254-257.

- Pansarasa, O., D. Rossi, A. Berardinelli, C. Cereda (2014) Amyotrophic lateral sclerosis and skeletal muscle: an update. Mol Neurobiol 49. 984-990.

Patzkowski, J.C., R.V. Blanck, J.G. Owens, J.M Wilken, K.L. Kirk, J.C. Wenke, J.R. Hsu (2012) Comparative effect of orthosis design on functional performance. J Bone Joint Surg Am 94: 507-515

Peixinho, C.C., N.S. Martins, L.F. de Oliveira, J.C. Machado (2014) Structural adaptations of rat lateral gastrocnemius muscle-tendon complex to a chronic stretching program and their quantification based on ultrasound biomicroscopy and optical microscopic images. Clin Biomech (Bristol, Avon) 29: 57-62.

Perez-Terzic, C., M.K. Childers (2014) Regenerative rehabilitation: a new future? Am J Phys Med Rehabil 93(11 suppl 3): S73-S78.

Ploutz, L.L., P.A. Tesch, R.L. Biro, G.A. Dudley (1994) Effect of resistance training on muscle use during exercise. J Appl Physiol (1985) 76: 1675-1681.

Rivera, J.C., B.T. Corona (2016) Muscle-related disability following combat injury increases with time. US Army Med Dep J, pp 30-34.

Rossi, C.A., M. Flaibani, B. Blaauw, M. Pozzobon, E. Figallo, C. Reggiani, L. Vitiello, N. Elvassore, P. De Coppi (2011) In vivo tissue engineering of functional skeletal muscle by freshly isolated satellite cells embedded in a photopolymerizable hydrogel. FASEB J 25: 2296-2304

Sandri, M., L. Barberi, A.Y. Bijlsma, B. Blaauw, K.A. Dyar, G. Milan, C. Mammucari, C.G. Meskers, G. Pallafacchina, A. Paoli, D. Pion, M. Roceri, V. Romanello, A.L. Serrano, L. Toniolo, L. Larsson, A.B. Maier, P. MunozCanoves, A. Musaro, M. Pende, C. Reggiani, R. Rizzuto, S. Schiaffino (2013) Signalling pathways regulating muscle mass in ageing skeletal muscle: the role of the IGF1-AktmTOR-FoxO pathway. Biogerontology 14: 303-323.

Schiaffino, S., V. Hanzlikova (1970) On the mechanism of compensatory hypertrophy in skeletal muscles. Experientia 26: 152-153.

Sicari, B.M., J.P. Rubin, C.L. Dearth, M.T. Wolf, F. Ambrosio, M. Boninger, N.J. Turner, D.J. Weber, T.W. Simpson, A. Wyse, E.H. Brown, J.L. Dziki, L.E. Fisher, S. Brown, S.F. Badylak (2014) An acellular biologic scaffold promotes skeletal muscle formation in mice and humans with volumetric muscle loss. Sci Transl Med 6: 234ra258.

Studitsky, A.N. (1964) Free auto- and homografts of muscle tissue in experiments on animals. Ann NY Acad Sci 120: 789-801.

Tamaki, T., S. Uchiyama, S. Nakano (1992) A weight-lifting exercise model for inducing hypertrophy in the hindlimb muscles of rats. Med Sci Sports Exerc 24: 881-886. 
Trappe, T. (2009) Influence of aging and longterm unloading on the structure and function of human skeletal muscle. Appl Physiol Nutr Metab 34: 459-464.

Turner, N.J., A.J. Yates, Jr., D.J. Weber, I.R Qureshi, D.B. Stolz, T.W. Gilbert, S.F. Badylak (2010) Xenogeneic extracellular matrix as an inductive scaffold for regeneration of a functioning musculotendinous junction. Tissue Eng Part A 16: 3309-3317.

Walters, T.J., K. Garg, B.T. Corona (2015) Activity attenuates skeletal muscle fiber damage after ischemia and reperfusion. Muscle Nerve 52: 640-648.

Walters, T.J., H.L. Sweeney, R.P. Farrar (1991) Influence of electrical stimulation on a fasttwitch muscle in aging rats. J Appl Physiol (1985) 71: 1921-1928.

Ward, C.L., L. Ji, B.T. Corona (2015) An autologous muscle tissue expansion approach for the treatment of volumetric muscle loss. BioRes Open Access 4: 198-208.
Warren, G.L., C.P. Ingalls, D.A. Lowe, R.B. Armstrong (2001) Excitation-contraction uncoupling: major role in contraction-induced muscle injury. Exerc Sport Sci Rev 29: 82-87.

Warren, G.L., A.L. Moran, H.A. Hogan, A.S. Lin, R.E. Guldberg, D.A. Lowe (2007) Voluntary run training but not estradiol deficiency alters the tibial bone-soleus muscle functional relationship in mice. Am J Physiol Regul Integr Comp Physiol 293: R2015-2026.

Warren, G.L., J.L. Stallone, M.R. Allen, S.A. Bloomfield (2004) Functional recovery of the plantarflexor muscle group after hindlimb unloading in the rat. Eur J Appl Physiol 93: 130-138.
Wenke, J.C., G.L. Warren, C.R. Rathbone, R.B. Armstrong (2010) Mouse plantar flexor muscle size and strength after inactivity and training. Aviat Space Environ Med 81: 632-638.

Willett, N.J., M.T. Li, B.A. Uhrig, J.D. Boerckel, N. Huebsch, T.L. Lundgren, G.L. Warren, R.E. Guldberg (2013) Attenuated human bone morphogenetic protein-2-mediated bone regeneration in a rat model of composite bone and muscle injury. Tissue Eng Part C Methods 19: 316-325.

Wolf, M.T., C.L. Dearth, S.B. Sonnenberg, E.G. Loboa, S.F. Badylak (2015) Naturally derived and synthetic scaffolds for skeletal muscle reconstruction. Adv Drug Deliv Rev 84: 208 221.

Wu, X., Corona, B.T., Chen, X., Walters, T.J. (2012) A standardized rat model of volumetric muscle loss injury for the development of tissue engineering therapies. BioRes Open Access 1: 280-290. 